\title{
"Hanging onto my joints": A qualitative study to explore the experience of osteonecrosis in teenagers and young adults living with and beyond cancer.
}

\author{
N. Chesman1, Dr L. McWilliams' ${ }^{1,2}$, Professor J. Yorke ${ }^{1,2}$ \\ 1The Christie NHS Foundation Trust, Manchester, United Kingdom. \\ 2University of Manchester, Manchester, United Kingdom.
}

\section{Background}

Osteonecrosis (ON) is a long-term side effect of cancer treatment in teenagers and young adults (TYAs, aged 16 to 24 years). It is a painful, disabling condition involving the irreversible death of bone tissue, leading to the degeneration and collapse of affected joints (1). The condition is more prevalent in TYAs than other age groups (2). Although the aetiology of ON in TYAs with cancer is not fully understood, it is agreed that the increasingly intensive use of corticosteroids in treatment protocols for young people, particularly for acute lymphoblastic leukaemia (ALL), is the most significant contributing factor (3).

Although there is evidence of ON in TYAs with a range of cancer diagnoses, available incidence rates come from studies of TYAs with ALL. The most recent UK figures suggest that $15.4 \%$ of those aged 16 to 24 years at the time of ALL diagnosis developed ON (4).

Osteonecrosis has no cure, and TYAs with the condition may require repeated joint replacements, of multiple joints, during their lifetime (5). However, little is known about the impact of ON on TYAs living with and beyond cancer.

Aim

To qualitatively explore the experience of ON in TYAs living with and beyond cancer.

\section{Methods}

This single-centre study was based at a specialist centre for TYAs with cancer in England. Eligible individuals were those who had received (or were continuing to receive) treatment for any form of cancer between the ages of 16 and 24 years; had been diagnosed with ON of any anatomical region; and were aged between 16 and 30 years at the time of recruitment. Participants were recruited via staff at the specialist centre and advertisements in clinical areas. Data were collected via semi-structured interviews, and analysed using thematic analysis.

\section{Results}

Eight young people participated in the study, including six males and two females. The mean age of participants at the time of interview was 25.5 years (range 18 to 30 years). All participants had been diagnosed with ALL, and had been living with ON from less than a year to over 10 years. The number of locations of ON in each participant ranged from two (for example, bilateral shoulders) to 12 (bilateral shoulders, elbows, wrists, hips, knees and ankles).

All participants found living with ON to be an entirely negative experience. Thematic analysis of interview data produced four themes, described below.

\section{Loss}

Pain and restricted range of movement caused losses across physical and social aspects of participants' lives. Participants struggled to complete essential tasks such as walking, washing and dressing, as well as hobbies, exercise and looking after children. Some became reliant on others for such tasks.

"To have to ask your brother or your mum or dad or something to give you a hand, just getting dressed. It really is demoralising. For me it was embarrassing."

Participants also experienced a loss of identity, and some no longer felt like a "normal" young person.

\section{Juggling act}

Living with $\mathrm{ON}$ in a context of loss and uncertainty involved a constant "juggling act". This included both the longer-term juggling act of "hanging onto my joints", and the daily balancing act of self-management.

"The doctors said to me "[Joint replacement is] the only option you have, so how long

can you go until you're in so much pain where you need it done straight away?". So from that point I held off for maybe two years [...] and then I was like "I can't do it, I literally can't do it anymore, I need to get [the replacements] done."

"You just have to weigh up what's worse really. Like, is [taking analgesics] worth it cos you know you're going to feel rubbish from the painkillers later. But if l'm up all night with pain l'm gonna be exhausted the next day anyway. So you have to kind of weigh up what is the lesser of two evils."

Distinct from the losses caused by physical inability, these juggling acts reflect the conscious "trade-offs" made by participants in the short- and long-term to attempt to manage and control their ON.
Uncertainty

Most participants were unaware of their risk of developing ON prior to being diagnosed with it. Participants received little information about ON, felt unprepared for living with it and unsure of who to turn to for advice and treatment.

"There's generally not a lot of awareness between clinicians on how to treat [ON] or how to help you. So it just feels like you're dealing with [ON] yourself and by yourself, and there's no one out there to help you."

Lack of information about the prognosis of ON, plus the potential requirement for repeat joint replacements, made participants uncertain as to their future physical abilities.

"[The ON] might not get any worse, and I might feel fine, but it might get worse. It's

just, I don't know do I? That's all it is, it's the not knowing."

"I know as you get older [joint replacement surgery] becomes harder, and when you do it over and over again it becomes harder, the repeat surgeries. It is quite scary to be honest. I might get to the point where [doctors] might say my body can't take [another joint replacement] .....and I might have to sacrifice my mobility."

\section{Conclusion}

This study is the first to explore the experience of ON in TYAs living with and beyond cancer. Findings suggest that ON has a wide-ranging, negative impact, producing additional challenges and uncertainties in the context of lives already disrupted by cancer. Future research into multiple aspects of cancer-related ON in TYAs is required, in addition to improvements in the information and support provided to those with the condition.

\section{References}

1. Schmiegelow, K., Attarbasschi, A., Barzilai, S., Escherich, G., Frandsen, T., Halsey, C., Hough, R., Jeha, S., Kato, M., Liang, D-C., Mikkelsen, T., Möricke, A., Niinimäki, R., Piette, C., Putti, M., Raetz, E., Silverman, L., Skinner, R., Tuckuviene, R., van der Sluis, I., Zapotocka, E. (2016). Consensus definitions of 14 severe acute toxic effects for childhood lymphoblastic leukaemia treatment: A Delphi consensus. Lancet Oncology, 17 (6), e231-e239.

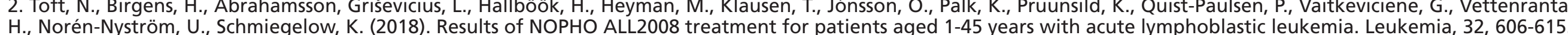

